\title{
Comparação entre dois sistemas de treino de força no desenvolvimento da força muscular máxima
}

\author{
Comparison between two strength training systems on the maximum \\ muscular strength performance
}

\author{
W. Materko, M. Duarte, E.L. Santos, H.S. Junior
}

\begin{abstract}
RESUMO
A proposta do presente estudo foi comparar os sistemas de treino tradicional (ST) e piramidal (SP) ao longo de oito semanas de treino sobre o desenvolvimento da força muscular máxima. Participaram dezoito homens experientes em treino de força, divididos em dois grupos de nove voluntários. O grupo tradicional seguiu um treino de 3 sets de 8 repetições ( $75 \%$ de 1RM) e grupo piramidal, 3 sets de 10, 8 e 6 repetições (70, 75 e 80\% de 1RM, respectivamente), com frequência de 4 vezes por semana. Estes passaram por uma avaliação antropométrica seguida de um teste de 1RM nos exercícios supino reto e agachamento. O teste de 1RM foi repetido após oito semanas de treino. Para analisar a significância dos resultados 1RM entre os sistemas, utilizou-se o teste de Mann-Whitney e, finalmente, o teste Wilcoxon para amostras pares foi usado para comparar os períodos pré e pós-treino. Não se registaram diferenças significativas entre os sistemas ST e SP no supino reto $(125 \pm 19 \mathrm{~kg}$ e $120 \pm 17 \mathrm{~kg})$ e agachamento $(124 \pm 18 \mathrm{~kg}$ e $120 \pm 17 \mathrm{~kg}$ ), assim como, também não registou diferença significativa entre os períodos pré e pós-treino. Em conclusão, os resultados sugerem que os sistemas de treino não geram diferenças no desempenho da força muscular máxima.
\end{abstract}

Palavras-chave: treino de força, força muscular, teste de 1RM

ABSTRACT

The goal of the present study was to compare traditional (ST) and pyramid (SP) strength training systems during eight weeks on the maximum muscular strength performance. Eighteen experienced in strength training men were divided into two groups of nine volunteers. Four times a week, the ST group trained in three sets of eight repetitions (75\% of 1RM) and the SP group in three sets of 10 , eight and six repetitions (70\%, $75 \%$ and $80 \%$ of 1 RM, respectively). All subjects were submitted to an anthropometric evaluation, followed by $1 \mathrm{RM}$ test in the bench press and squat exercises, which were repeated after eight weeks of training. The difference between the attained 1RM for each system was studied using Mann-Whitney test and the Wilcoxon paired test was applied to compare pre- and posttraining. No significant differences were recorded between ST and SP bench press (125 $\pm 19 \mathrm{~kg}$ and $120 \pm 17.0 \mathrm{~kg})$ and squat $(124 \pm 18 \mathrm{~kg}$ and $120 \pm 17 \mathrm{~kg})$. Furthermore, no significant differences were found between the pre- and post-training periods. According to the present results, both training systems produced similar effects on the maximum muscular strength performance.

Keywords: strength training, muscular strength, test of 1RM

Submetido: 13.09.2009 | Aceite: 23.01.2010

Wollner Materko, Marcelo Duarte e Homero da Silva Junior. Laboratório de Pesquisa em Fisiologia do Exercício, Curso de Educação Física, Universidade Estácio de Sá, Campus Rebouças, Rio de Janeiro, Brasil.

Edil Luis Santos. Programa de Engenharia Biomédica, COPPE - Universidade Federal do Rio de Janeiro, Brasil. Endereço para correspondência: Wollner Materko, Universidade Estácio de Sá, Rua Cândido Mendes, nº140, apt. 805, Glória, CEP: 20241-220 Rio de Janeiro, RJ, Brasil.

E-mail: wollner.materko@gmail.com 
O treino de força tem sido apontado em inúmeros estudos (Aagaard, Simonsen, Andersen, Magnusson, \& Dyhre-Poulsen, 2002; Ades et al., 2005; Kraemer \& Ratamess, 2004) como método efetivo para o aumento de força e hipertrofia muscular, além de proporcionar aumento da densidade óssea (Brentano et al., 2008), poder reduzir os fatores de risco associados à doença arterial coronariana (Ades et al., 2005), diabetes Mellitus tipo II (Shaibi et al., 2006), cancro de cólon (Schmitz, Ahmed, \& Yee, 2002), controle da obesidade (Leick et al., 2007) e no envelhecimento (Costa \& Fernandes, 2007).

É comumente estabelecido que a prescrição de exercício em treino de força através da relação entre o percentual de 1RM e o número de repetições (Moore et al., 2004). Os benefícios e adaptações decorrentes deste tipo de exercício são decorrentes de uma série de variáveis, tais como número de séries (Galvão \& Taaffe, 2004), número de repetições (Shimano et al., 2006), frequência semanal (Burt, Wilson, \& Willardson, 2007), tempo de intervalo entre as séries e sessões de treino (Willardson \& Burkett, 2008), intensidade e volume de treino (Prestes, De Lima, Frollini, Donatto, \& Conte, 2009), tipo de acções musculares (Blazevich, Cannavan, Coleman, \& Horne, 2007), ordem dos exercícios (Gentil, Oliveira, De Araújo, Do Carmo, \& Bottaro, 2007) e velocidade de movimento (Wickwire, McLester, Green, \& Crews, 2009). Com isso, o treinador ou preparador físico, conhecendo os diferentes tipos de treino, pode otimizar o condicionamento físico de seus atletas para determinada modalidade desportiva (Carpinelli, Otto, \& Winett, 2004; Peterson, Rhea, \& Alvar, 2005).

Fleck e Kraemer (1999) afirmam que os sistemas de treino de força foram originalmente planejados por levantadores olímpicos de peso ou fisiculturistas, entre os quais temos o sistema de tradicional (ST) e o sistema piramidal (SP). Estes são realizados de forma empírica para melhoria de resultado em força, potência ou hipertrofia muscular, portanto, são necessários mais estudos sobre os sistemas de treino de força muscular, pois está diretamente relacionado ao quotidiano de diversos profissionais de Educação Física em academias ou ginásios, e principalmente, porque os praticantes concluem de facto que um sistema funcione melhor do que outro. Actualmente, até o nosso conhecimento, nenhum estudo comparou o aumento da força muscular entre os sistemas tradicional e o piramidal durante treino de força muscular. Sendo assim, o objectivo do presente estudo foi avaliar o efeito dos sistemas de treino tradicional e o sistema piramidal ao longo de oito semanas de treino sobre o desenvolvimento da força muscular máxima.

\section{MÉTODO}

\section{Amostra}

Participaram deste estudo 18 voluntários do sexo masculino, selecionados aleatoriamente em uma academia de ginástica do município do Rio de Janeiro. Consideraram-se como critérios de elegibilidade: que os voluntários tivessem, no mínimo, seis meses de experiência em treino de força muscular, que não utilizassem qualquer recurso ergorgênico e que não apresentassem lesões osteomioarticulares prévias. Estes foram classificados como indivíduos de baixo risco, por apresentarem até um fator de risco para doença arterial coronariana e não apresentarem qualquer sinal ou sintoma sugestivo de doença cardiopulmonar ou metabólica.

Todos foram previamente instruídos a não realizar exercício físico nas $24 \mathrm{~h}$ precedentes, não consumir bebida alcoólica e a manterem-se hidratados ao longo dos testes. Os procedimentos experimentais tiveram início somente após o consentimento verbal e a assinatura do termo de consentimento livre e esclarecido, conforme aprovado pelo Comité de Ética em Pesquisa da Instituição e todos os procedimentos utilizados respeitaram a Declaração de Helsinque de 1975. Os voluntários foram inicialmente submetidos a uma avaliação antropométrica, seguida de um 
teste de 1RM de familarização (Ploutz-Snyder \& Giamis, 2001), e obedecendo a um intervalo mínimo de $48 \mathrm{~h}$, realizaram um re-teste de 1RM. Todos os testes de 1RM foram realizados entre 17:00 e 19:00 horas. Nos intervalos entre as sessões de testes não foi permitida a realização de exercícios, para não interferir nos resultados obtidos.

\section{Instrumentos}

\section{Avaliação antropométrica}

Esta constou da medida de massa corporal e estatura, realizada numa balança mecânica com estadiômetro (Filizola, Brasil) e para tomada das medidas das sete dobras cutâneas seguiram as técnicas descritas por Lohman (1992) através de um compasso científico (Cescorf, Brasil). A partir destas medidas, calculou-se o percentual de gordura e a massa livre de gordura usando as equações de Jackson e Pollock (1978) para a estimativa da densidade corporal em homens, respectivamente, combinada com a equação de Siri (1961).

\section{Protocolo do teste de 1RM}

Os testes de 1RM foram conduzidos conforme o protocolo proposto por Brown e Weir (2001). Realizaram-se três a cinco minutos de atividades leves envolvendo o grupamento muscular testado, e após um minuto de alongamento leve, aquecimento de oito repetições a $50 \%$ de 1RM estimada pelo avaliador e seguido de três repetições a $70 \%$ de 1RM estimada pelo avaliador. Após cinco minutos de intervalo, realizou-se o teste de 1RM, acrescentando-se, quando necessário, .5 a $5.0 \mathrm{~kg}$, totalizando três a cinco tentativas. Registrou-se como carga máxima aquela levantada em único movimento. Todos os testes de 1RM foram conduzidos em um equipamento Smith machine (Buick Industries, Brazil). Selecionaram-se os exercícios de supino reto e agachamento pelo facto de se tratar de um movimento pluriarticular, além de oferecerem um baixo risco de lesão.

\section{Procedimentos}

\section{Teste Supino Reto}

O teste partiu da posição inicial em decúbito dorsal no banco, joelhos flexionados, com os pés sobre banco, cotovelos estendidos, ombros aduzidos horizontalmente e a pegada na barra para cada avaliado foi padronizado a partir do afastamento das mãos na largura dos ombros. A execução teve início com a fase excêntrica limitado com os braços paralelos ao solo, na fase concêntrica realizou-se a adução horizontal de ombros e extensão de cotovelos simultaneamente retornando à posição inicial.

\section{Teste Agachamento}

O teste partiu da posição inicial em pé com a barra apoiada nas costas, com os pés paralelos e as articulações do joelho e do quadril em extensão total. A execução teve início com a fase excêntrica limitado com as coxas paralelas ao solo, na fase concêntrica realizou-se a extensão de quadril e extensão de joelho simultaneamente retornando à posição inicial.

\section{Protocolo de Treino}

Após a obtenção das cargas em 1RM, a inclusão dos indivíduos nos grupos foi definida, alternadamente, pela técnica da aleatoriedade em dois grupos de nove voluntários denominados: (1) grupo tradicional (GT) com a execução de 3 sets de 8 repetições a $75 \%$ de $1 \mathrm{RM}$; e (2) grupo piramidal (GP) com a execução de 3 sets de 10, 8 e 6 repetições a 70, 75 e $80 \%$ de 1RM, respectivamente. Cada grupo realizou a sequência de exercícios estipulada em duas formas (tabela 1), treinando quatro vezes por semana (segunda, terça, quinta e sexta), sendo segunda e quinta, a sequência $A$, na qual eram trabalhados exercícios para peitoral, tríceps braquial e abdômen; e, terça e sexta, a sequência $B$, que consistia em exercícios para a região dorsal, bíceps braquial e membros inferiores. Foram realizadas oito semanas de treino com quatro sessões semanais. $\mathrm{O}$ intervalo entre as séries foi de 2 minutos e 48 horas de intervalo entre as sequências e não foi 
acrescentada sobrecarga, como progressão do treino. Antes de realizar o primeiro exercício na sequência adotada, foram realizadas 12 repetições com $40 \%$ de $1 \mathrm{RM}$ como forma de aquecimento. Após oito semanas de treino, realizaram novamente os testes de $1 \mathrm{RM}$ no exercício supino reto e agachamento para comparar o desenvolvimento da força muscular máxima (1RM) entre os métodos de treino e no instante pré e pós-treino.

Tabela 1

Sequências de exercícios durante oito semanas de treino

\begin{tabular}{cc}
\hline Sequência A & Sequência B \\
\hline 1 - Supino no Smith & 1 - Pulley puxada pela frente \\
2 - Supino inclinado & 2 - Remada sentada pegada fechada \\
3 - Voador frontal & 3 - Voador dorsal \\
4 - Desenvolvimento na máquina pegada aberta & 4 - Bíceps alternado \\
5 - Tríceps testa & 5 - Bíceps no cabo \\
6 - Tríceps no cabo & 6 - Agachamento no Smith \\
7 - Flexão parcial de tronco & 7 - Leg press $45^{\circ}$ \\
& 8 - Flexão plantar na máquina \\
\hline
\end{tabular}

\section{Análise Estatística}

Para determinar a normalidade da distribuição, utilizou-se o teste Kolmogorov-Smirnov, verificando-se que a amostra não seguiu uma distribuição gaussiana. A análise estatística dividiu-se em descritiva e inferencial. A primeira buscou a definição do perfil dos grupos, sendo expressa como média e desvio padrão, enquanto a segunda buscou comparálos (inter e intragrupos). Com isso, utilizou-se o teste não paramétrico de Mann-Whitney para analisar a significância intergrupo dos resultados comparativos dos sistemas de treino e utilizou-se também o teste não paramétrico de pares ordenados de Wilcoxon para comparar intragrupo a diferença entre os períodos pré e pós-treino. Adotou-se em todos os testes empregados um valor de $p<.05$. Todos os procedimentos estatísticos foram realizados em Statistica v.8 (Statsoft, EUA).

\section{RESULTADOS}

A tabela 2 apresenta as características físicas e antropométricas, cuja baixa dispersão dos dados aponta para um grupo de voluntário bastante homogêneo, apesar da distribuição não apresentar normalidade.
Não se registrou nenhum problema osteomioarticular durante ou imediatamente após qualquer teste, de forma que todos os voluntários chegaram à carga máxima nos testes de 1RM em ambos os exercícios.

Tabela 2

Características físicas e antropométricas dos voluntários

\begin{tabular}{cc}
\hline Variáveis & Média \pm DP \\
\hline Idade (anos) & $23.0 \pm 3.0$ \\
Massa corporal $(\mathrm{kg})$ & $76.7 \pm 7.0$ \\
Estatura $(\mathrm{cm})$ & $175.3 \pm 5.5$ \\
Gordura relativa $(\%)$ & $12.0 \pm 4.0$ \\
\hline
\end{tabular}

Portanto, tal qual exposto na tabela 3, demonstra-se a análise de significância do teste de Mann-Whitney do resultado comparativo nos sistemas de treino apresentando em média $125 \pm 19 \mathrm{~kg}$ (GP) e $120 \pm 17 \mathrm{~kg}$ (GT), constatando-se que a diferença não foi significativa para o teste de 1RM no exercício supino reto. Similarmente, o exercício agachamento resultou em $124 \pm 18 \mathrm{~kg}$ (GP) e $120 \pm 17 \mathrm{~kg}$ (GT). Apesar da tendência do aumento da carga de 1RM do GP em relação ao GT, não apresentaram diferenças significativas para ambos exercícios $(p>.05)$. 
Tabela 3

Resultados comparativos entre os sistemas de treino na força muscular máxima (1RM)

\begin{tabular}{cccl}
\hline Exercícios & Sistema Piramidal & Sistema Tradicional & $p$ \\
\hline Supino reto $(\mathrm{kg})$ & $125 \pm 19$ & $120 \pm 17$ & .38 \\
Agachamento $(\mathrm{kg})$ & $124 \pm 18$ & $120 \pm 17$ & .56 \\
\hline
\end{tabular}

Finalmente, a análise dos dados do teste de Wilcoxon para amostras pares, que permitiu comparar a diferença entre os períodos pré e pós-treino (tabela 4), demonstrou que os sistemas de treino GP e GT não resultaram em diferenças significativas para os testes de $1 \mathrm{RM}$ no exercício supino reto e agachamento. No supino reto apresentou uma diferença média de $8 \mathrm{~kg}$ ou equivalente a $6.4 \%$ do teste de $1 \mathrm{RM}$ para o GP e uma diferença média de $7 \mathrm{~kg}$ ou equivalente a $5 \%$ do teste de $1 \mathrm{RM}$ para o GT, tal como, o exercício agachamento apresentou uma diferença média de $5 \mathrm{~kg}$ ou equivalente a $4 \%$ do teste de 1 RM para o GP e uma diferença média de $7 \mathrm{~kg}$ ou equivalente a $6 \%$ do teste de 1RM para o GT.

Tabela 4

Resultados entre os períodos pré e pós-treino na força muscular máxima (1RM)

\begin{tabular}{cccccc}
\hline \multirow{2}{*}{ Exercícios } & \multicolumn{2}{c}{ Sistema Piramidal } & \multicolumn{2}{c}{ Sistema Tradicional } & $p$ \\
& Pré & Pós & Pré & Pós & \\
\hline Supino reto $(\mathrm{kg})$ & $117 \pm 18$ & $125 \pm 19$ & $112 \pm 17$ & $120 \pm 17$ & .07 \\
Agachamento $(\mathrm{kg})$ & $119 \pm 17$ & $124 \pm 18$ & $113 \pm 18$ & $120 \pm 17$ & .07 \\
\hline
\end{tabular}

\section{DISCUSSÃO}

O presente estudo avaliou o efeito dos sistemas de treino tradicional e o sistema piramidal ao longo de oito semanas de treino sobre o desenvolvimento da força muscular máxima, na qual, não se registrou diferença significativa entre os sistemas de treino tradicional e piramidal ao longo de oito semanas de treino de força e nem entre os períodos pré e pós-treino, em relação à força máxima dinâmica em ambos os exercícios, apesar da média do incremento de carga no sistema de treino piramidal ter sido maior, o aumento da carga em ambos os sistemas entre o período pré e pós-teste foram similares nos exercícios investigados. Além disso, até o nosso conhecimento nenhum estudo comparou o aumento da força muscular entre os sistemas tradicional e o piramidal durante o treino de força muscular.

Apesar da falta de estudo sobre os diferentes tipos de sistemas de treino, um estudo recente (Salles, Silva, Oliveira, Ribeiro, \& Simão, 2008) comparou o sistema piramidal crescente e decrescente sobre o número total de repetições máximas em uma sessão de treino de força em catorze indivíduos experientes em treino de força muscular. Todavia, não foi encontrada diferença entre as repetições máximas totais realizadas nos dois sistemas de treino. Este resultado corrobora com os do presente estudo, apesar dos sistemas de treino serem diferentes.

O treino de força dinâmico de alta intensidade está relacionado a uma elevada exigência do sistema nervoso em solicitar uma maior solicitação e frequência de disparo das unidades motoras, consequentemente, aumento na força muscular (Nosaka \& Newton, 2002). O tempo exato para que ocorra um aumento da força máxima, assim como, hipertrofia muscular significativa é divergente na literatura, mas parece que oito semanas de treino de força muscular numa frequência de três vezes na semana já é suficiente para aumento da carga de 1RM para membros inferiores (Ibánez et al., 2008) e membros superiores (Moore et al., 2004), bem como 
para a potência do salto vertical (Winchester et al., 2008).

Kravitz, Akalan, Nowicki e Kinzey (2003) estudaram jovens atletas de levantamento de peso, o tempo de experiência foi mais bem correlacionado $(r=.70)$ à 1RM que qualquer outra variável antropométrica, isto demonstra como esta variável tem grande influência no desenvolvimento da força muscular. Em estudos recentes, demonstraram que o tempo de experiência no mínimo de seis meses em treino de força influenciou na predição na carga de 1RM (Materko, Neves, \& Santos, 2007; Materko \& Santos, 2009) e um estudo clássico na literatura (Moritani \& De Vries, 1979), observou que o aumento agudo na força muscular é ocasionado pela aprendizagem motora, isto devido à maior frequência de descarga e pelo aumento no recrutamento das unidades motoras. Portanto, a experiência em treino de força pode ser uma variável interveniente no resultado do presente estudo, pois ambos os resultados não apresentaram diferenças significativas entre os sistemas de treino tradicional e piramidal e entre os períodos pré e pós-treino para os testes de 1RM em ambos os exercícios.

Além disso, estudos anteriores têm mostrado que inúmeros fatores - como o nível de condicionamento físico (Hoeger, Hopkins, Barette, \& Hale, 1990), o grupamento muscular (Hoeger, Barette, Hale, \& Hopkins, 1987), sono (Blumert et al., 2007), alimentação (Degoutte et al., 2006), ritmo cronobiológico (Atkison \& Reilly, 1996), motivação (Fry \& Fry, 1999), recurso ergogênico (Materko, Novaes, \& Santos, 2008) e a fadiga muscular (Willardson, 2007) interferem efetivamente na relação do ganho da força muscular, resultando em distintas intensidades para dado número de repetições, talvez, essas variáveis intervenientes influenciaram negativamente no resultado do presente estudo.

Estudos anteriores (Aagaard et al., 2002; Petrofsky \& Laymon, 2004), em períodos a partir de oito semanas de treino de força são suficientes para provocar ganho na força muscular, porém, em indivíduos experientes em treino de força o ganho de força máxima dinâmica parece exigir um tempo maior de treino devido à adaptação neural do treino (Brill, Macera, Davis, Blair, \& Gordon, 2000), por isso, podemos observar que períodos até oito semanas de treino são insuficientes para provocar um aumento na força muscular em indivíduos treinados.

Buford, Rossi, Smith e Warren (2007) e Rhea et al (2003) observaram que dois diferentes métodos de treino de força muscular, um com periodização linear e outro com periodização ondulatória entre nove e quinze semanas com igual volume e intensidade, respectivamente, não apresentaram diferenças significativas na força muscular entre os métodos estudados. De acordo com o presente estudo, o não incremento de carga adicional durante $o$ período estudado, pôde ter sido fundamental para os resultados não serem significativos, apesar de estes não avaliaram a força muscular máxima tal verificando no presente estudo.

Chilibeck, Calder, Sale e Webber (1998) estudaram o efeito do treino de força muscular durante vinte semanas em dezanove mulheres com experiência em TCR, com objectivo de comparar o ganho de força nos exercícios supino reto, leg press e rosca bíceps, com isso, observou-se uma maior adaptação neural para os exercícios de maior complexidade como o supino reto e leg press e, consequentemente, um maior ganho na força muscular para o exercício rosca bíceps, pois a adaptação neural foi mais rápida. Portanto, hipoteticamente, se o presente estudo testasse exercícios de menor complexidade, talvez poderia ter um resultado diferente.

Cabe apontar que o presente restringe-se a homens experientes em treino de força, em exercícios pluriarticulares para avaliação dos sistemas de treino, um período determinado de oito semanas e sem progressão da sobrecarga de treino. Talvez, o incremento de carga nos exercícios em ambos os sistemas durante o 
período de oito semanas poderia trazer resultados significativos.

Portanto, recomendam-se outras pesquisas relacionando sistemas de treino de força, principalmente, em indivíduos com e sem experiência em treino de força muscular, ambos os sexos, diferentes períodos de treino e progressão da sobrecarga de treino.

\section{REFERÊNCIAS}

Aagaard, P., Simonsen, E. B., Andersen, J. L., Magnusson, P., \& Dyhre-Poulsen, P. (2002). Increased rate of force development and neural drive of human skeletal muscle following resistance training. Journal of Applied Physiology, 93, 1318-1326.

Ades, P. A., Savage, P. D., Brochu, M., Tischler, M. D., Lee, N. M., \& Poehlman, E. T. (2005). Resistance training increases total daily energy expenditure in disabled older women with coronary heart disease. Journal of Applied Physiology, 98(4), 1280-1285.

Ahtiainen, J. P., Pakarinen, A., Alen, M., Kraemer, W. J., \& Häkkinen, K. (2003). Muscle hypertrophy, hormonal adaptations and strength development during strength training in strength-trained and untrained men. European Journal Applied Physiology, 89, 555-563.

Atkison, G., \& Reilly, T. (1996). Circadian variation in sports performance. Sports Medicine, 36, 292312.

Blazevich, A. J., Cannavan, D., Coleman, D. R., \& Horne, S. (2007). Influence of concentric and eccentric resistance training on architectural adaptation in human quadriceps muscles. Journal of Applied Physiology, 103 (5), 1565-1575.

Blumert, P. A., Crum, A. J., Ernsting, M., Volek, J. S., Hollander, D. B., Haff, E. E., et al. (2007). The acute effects of twenty-four of sleep loss on the performance of national-caliber male collegiate weightlifters. Journal of Strength and Conditioning Research, 21 (4), 1146-1154.

Brentano, M. A., Cadore, E. L., Da Silva, E. M., Ambrosini, A. B., Coertjens, M., Petkowicz, R., et al. (2008). Physiological adaptations to strength and circuit training in postmenopausal women with bone loss. Journal of Strength and Conditioning Research, 22(6), 1816-1825.

Brill, P. A., Macera, C. A., Davis, D. R., Blair, S. N., \& Gordon, N. (2000). Muscular strength physical function. Medicine and Science in Sports and Exercise, 32(2), 412-416.

Brown, L. E., \& Weir, J. P. (2001). ASEP Procedures recommendation I: Accurate assessment of muscular strength and power. Journal of Exercise Physiology - online, 4, 1-21.

Buford, T. W., Rossi, S. J., Smith, D. B., \& Warren, A. J. (2007). A comparison of periodization models during nine weeks with equated volume and intensity for strength. Journal of Strength and Conditioning Research, 21(4), 12451250.

Burt, J., Wilson, R., \& Willardson, J. M. (2007). A comparison of once versus twice per week training on leg press strength in women. The Journal of Sports Medicine and Physical Fitness, 47(1), 13-17.

Carpinelli, R. N., Otto, R. M., \& Winett, R. A. (2004). A critical analysis of the ACSM position stand on resistance training: Insufficient evidence to support recommended training protocols. Journal of Exercise Physiology online, 7(3), 1-60.

Chilibeck, P. D., Calder, A. W., Sale, D. G., \& Webber, C. E. (1998). A comparison of strength and muscle mass increases during resistance training in young women. European Journal of Applied Physiology and Occupational Physiology, 77(1-2), 170-175.

Costa, A., \& Fernandes, C. (2007). Utilização da percepção subjectiva do esforço para monitorização da intensidade do treino de força em Idosos. Revista Motricidade, 3(2), 37-46.

Degoutte, F., Jouanel, P., Bègue, R. J., Colombier, M., Lac, G., Pequignot, J. M., et al. (2006). Food restriction, performance, biochemical, psychological and endocrine changes in judo athletes. International Journal of Sports Medicine, 27(1), 9-18.

Fleck, S. J., \& Kraemer, W. J. (1999). Fundamentos do treinamento de força muscular ( $2^{\mathrm{a}}$ ed). Porto Alegre: Artmed.

Fry, M. D., \& Fry, A. C. (1999). Goal perspectives and motivational responses of elite junior weightlifters. Journal of Strength and Conditioning Research, 13, 311-317.

Galvão, D. A., \& Taaffe, D. R. (2004). Single- vs. multiple-set resistance training: Recent developments in the controversy. Journal of Strength and Conditioning Research, 18(3), 660667. 
Gentil, P., Oliveira, E., De Araújo, R. J. V., Do Carmo, J., \& Bottaro, M. (2007). Effects of exercise order on upper-body muscle activation and exercise performance. Journal of Strength and Conditioning Research, 21 (4), 1082-1086.

Hoeger, W. W. K., Barette, S. L., Hale, D. F., \& Hopkins, D. R. (1987). Relationship between repetitions and selected percentages of one repetition maximum. Journal of Applied Sport Science Research, 1, 11-13.

Hoeger, W. W. K., Hopkins, D. R., Barette, S. L., \& Hale, D. F. (1990). Relationship between repetitions and selected percentages of one repetition maximum: A comparison between untrained and trained males and females. Journal of Applied Sport Science Research, 4, 47-54.

Ibánez, J., Gorostiaga, E. M., Alonso, A. M., Forga, L., Argüelles, I., Larrión, J. L., et al. (2008). Lower muscle strength gains in older men with type 2 diabetes after resistance training. Journal of Diabetes and Its Complications, 22 (2), 112-118.

Jackson, A. S., \& Pollock, M. (1978). Generalized equations for predicting body density of men. The British Journal of Nutrition, 40, 497-504.

Kraemer, W. J., \& Ratamess, N. A. (2004). Fundamentals of resistance training: Progression and exercise prescription. Medicine and Science in Sports and Exercise, 36 (4), 674-688.

Kravitz, L., Akalan, C., Nowicki, K., \& Kinzey, S. J. (2003). Prediction of 1 repetition maximum in high-school power lifters. Journal of Strength and Conditioning Research, 17, 167-172.

Leick, L., Lindegaard, B., Stensvold, D., Plomgaard, P., Saltin, B., \& Pilegaard, H. (2007). Adipose tissue interleukin-18 mRNA and plasma interleukin-18: Effect of obesity and exercise. Obesity (Silver Spring), 15(2), 356-363.

Lohman, T. G. (1992). Advances in body composition assessment. Champaign, IL: Human Kinetics.

Materko, W., Neves, C. E. B., \& Santos, E. L. (2007). Prediction model of a maximal repetition (1RM) based on male and female anthropometrical characteristics. Revista Brasileira de Medicina do Esporte, 13(1), 27-31.

Materko, W., Novaes, J. S., \& Santos, E. L. (2008). Effect of bicarbonate supplementation on the muscular strength. Journal of Exercise Physiology online, $11(6), 25-33$.

Materko, W., \& Santos, E. L. (2009). Prediction of one repetition maximum (1RM) based on a submaximal strength test in adult males. Isokinetics and Exercise Science, 17(4), 189-195.
Moore, D. R., Burgomaster, K. A., Schofield, L. M., Gibala, M. J., Sale, D. G., \& Phillips, S. M. (2004). Neuromuscular adaptations in human muscle following low intensity resistance training with vascular occlusion. European Journal of Applied Physiology, 92 (4/5), 399-406.

Morales, J., \& Sobonya, S. (1996). Use of submaximal repetition tests for predicting $1 \mathrm{rm}$ strength in class athletes. Journal of Strength and Conditioning Research, 10, 186-189.

Moritani, T., \& De Vries, H. A. (1979). Neural factors versus hypertrophy in the time course of muscle strength gain. American Journal of Physical Medicine, 58, 115-130.

Nosaka, K., \& Newton, M. (2002). Concentric or eccentric training effect on eccentric exerciseinduced muscle damage. Medicine and Science in Sports Exercise, 34(1), 63-69.

Peterson, M. D., Rhea, M. R., \& Alvar, B. A. (2005). Applications of the dose-response for muscular strength development: A review of metaanalytic efficacy and reliability for designing training prescription. Journal of Strength and Conditioning Research, 19(4), 950-958.

Petrofsky, J. S., \& Laymon, M. (2004). The effect of previous weight training and concurrent weight training on endurance for functional electrical stimulation cycle ergometry. European Journal of Applied Physiology, 91, 392-398.

Ploutz-Snyder, L. L., \& Giamis, E. L. (2001). Orientation and familiarization to 1RM strength testing in old and young women. Journal of Strength and Conditioning Research, 15, 519-523.

Prestes, J., De Lima, C., Frollini, A. B., Donatto, F. F., \& Conte, M. (2009). Comparison of linear and reverse linear periodization effects on maximal strength and body composition. Journal of Strength and Conditioning Research, 23 (1), 266-274.

Rhea, M. R., Phillips, W. T., Burkett, L. N., Stone, W. J., Ball, S. D., Alvar, B. A., et al. (2003). A comparison of linear and daily undulating periodized programs with equated volume and intensity for local muscular endurance. Journal of Strength and Conditioning Research, 17(1), 8287.

Salles, B. F., Silva, J. P. M., Oliveira, D., Ribeiro, F. M., \& Simão, R. (2008). Efeito dos métodos pirâmide crescente e pirâmide decrescente no número de repetições do treinamento de força. Revista Arquivos em Movimentos, 4(1), 24-32. 
Schmitz, K. H., Ahmed, R. L., \& Yee, D. (2002). Effects of a 9-month strength training intervention on insulin, insulin-like growth factor (IGF)-I, IGF-binding protein (IGFBP)-1, and IGFBP-3 in 30-50-year-old women. Cancer Epidemiology, Biomarkers \& Prevention, 11(12), 1597-1604.

Shaibi, G. Q., Cruz, M. L., Ball, G. D., Weigensberg, M. J., Salem, G. J., Crespo, N. C., et al. (2006). Effects of resistance training on insulin sensitivity in overweight Latino adolescent males. Medicine and Science in Sports and Exercise, 38(7), 1208-1215.

Shimano, T., Kraemer, W. J., Spiering, B. A., Volek, J. S., Hatfield, D. L., Silvestre, R., et al. (2006). Relationship between the number of repetitions and selected percentages of one repetition maximum in free weight exercises in trained and untrained men. Journal of Strength and Conditioning Research, 20(4), 819-823.

Siri, W. E. (1961). Body composition from fluid spaces and density: Analysis of methods. In J. Brozek \& A. Henschel (Eds.), Techniques for measuring body composition (pp. 223-244). Washington, DC: National Academic of Science.
Wickwire, P. J., McLester, J. R., Green, J. M., \& Crews, T. R. (2009). Acute heart rate, blood preassure, and RPE response during super slow vs. traditional machine resistance training protocols using small muscle group exercises. Journal of Strength and Conditioning Research, 23(1), 72-79.

Willardson, J. M. (2007). The application of training to failure in periodized multiple-set resistance exercise programs. Journal of Strength and Conditioning Research, 21 (2), 628-631.

Willardson, J. M, \& Burkett, L. N. (2008). The effect of different rest intervals between sets on volume components and strength gains. Journal of Strength and Conditioning Research, 22 (1), 146152.

Winchester, J. B., McBride, J. M., Maher, M. A., Mikat, R. P., Allen, B. K., Kline, D. E., et al. (2008). Eight weeks of ballistic exercise improves power independently of changes in strength and muscle fiber type expression. Journal of Strength and Conditioning Research, $22(6), 1728-1734$. 